\title{
ESTADÍA PRÁCTICA DE LA LICENCIATURA EN GASTRONOMÍA Y SU EVIDENCIA DE DESEMPEÑO PROFESIONAL
}

\section{PRACTICAL STAY OF THE DEGREE IN GASTRONOMY AND YOUR EVIDENCE OF PROFESSIONAL PERFORMANCE ${ }^{1}$}

\author{
Rosa Idania Náfate Arroyo ${ }^{2}$ \\ Benjamín Gutiérrez Gutiérrez ${ }^{3}$
}

\section{KEY WORDS}

Professional Training Gastronomy

Competencies Practical stay Performance

\begin{abstract}
The professional training of the Gastronomy graduates of the Technological University of

1 Revista Internacional de Educación y Aprendizaje, $X(X), 202 X$, ISSN 2255-453X ๔ Global Knowledge Academics, los autores. http://journals.epistemopolis.org/ educacion

2 Estudiante del Doctorado en Investigación e Innovación Educativa de la Facultad de Filosofía y Letras de la BUAP, con beca Conacyt no. 620011 Maestría en Educación Superior y Maestría en Administración, Licenciada en Administración de Empresas Turísticas, docente de la División de Gastronomía de la UTP, e-mail: idania.nafate@utpuebla. edu.mx. ORCID: https://orcid.org/0000-0002-4278-905X

3 Doctor en Educación, Maestría en Educación Superior y Licenciado en Enseñanzas de Lenguas Extranjeras. Ha participado en varios proyectos de diseño y evaluación curricular. Además colabora en proyectos nacionales e internacionales, e-mail: tutorbenjamin@hotmail.com. https:// orcid.org/0000-0003-2716-9108
\end{abstract}

Puebla (UTP), is perceived as an essential part in the development of their professional skills to solve the problems they demand in the workplace. Food and Beverage Establishments increasingly require graduates to develop their professional skills as a key to performance, in carrying out specific activities in the different work areas. It is essential to verify that the students, by obtaining their Practical Stay (EP), achieve autonomous levels of performance in real work situations 


\section{PALBARAS CLAVE}

\section{Formación Profesional Gastronomía \\ Competencias Estadía Práctica Desempeño}

\section{RESUMEN}

La formación profesional en la Licenciatura en Gastronomía de la Universidad Tecnológica de Puebla (UTP) es percibida como parte esencial en el desarrollo de sus competencias profesionales para resolver los problemas que demandan en el ámbito laboral. Los Establecimientos de Alimentos y Bebidas exigen cada vez más en la ejecución de las actividades o tareas específicas en las distintas áreas de trabajo. Por lo cual es indispensable comprobar y observar que el estudiantado logre durante su Estadía Practica (EP) alcanzar niveles de desempeño autónomos que favorezcan a las exigencias mismas de la organización.

\section{INTRODUCCIÓN}

Actualmente se reconoce que el aprendizaje ocurre en distintas formas y circunstancias, por lo que se trata de un proceso dinámico e inherente a la naturaleza humana que se desarrolla a lo largo de la vida. En particular, es fundamental que los y las estudiantes de un nivel superior realicen ejercicios profesionales a través de prácticas asignadas a diversas Unidades Productivas y Sociales (UPS) en áreas específicas a su perfil de egreso, con la ventaja de mejorar sus habilidades desarrolladas durante su proceso de enseñanza aprendizaje. Como referencia, es el caso del Subsistema de Universidades Tecnológicas (SUUTT) en México, que en su Modelo Educativo determina la movilización de saberes de los y las estudiantes que forman parte de la profesionalización que obtienen para resolver satisfactoriamente los retos y exigencias que se les presente directamente en un contexto laboral. Se considera, que uno de los roles del docente es cumplir con la demanda social, nuevos enfoques educativos, así como implementar el Modelo Pedagógico correspondiente a cada institución. Por lo que, se requiere de una transformación que le permita responder de forma oportuna a las exigencias del entorno local, nacional e internacional. Esto, requiere del desarrollo de competencias profesionales apropiadas durante el proceso de enseñanza aprendizaje para evidenciar los desempeños obtenidos.

Con base, al Modelo Educativo del SUUTT tales como: el docente motivador en el desarrollo de actividades de enseñanza aprendizaje y adquisición de competencias profesionales a través de la formulación de perfiles de desempeño que constituyen el elemento central de los contenidos educativos, fomentando a cada estudiante la responsabilidad de tomar decisiones asertivas, considerar un pensamiento crítico -reflexivo, según sus posibilidades y grado de madurez, así como fomentar la socialización de los estudiantes con relación al trabajo colaborativo, desarrollo de proyectos, participación en eventos institucionales, asistencia a congresos y visitas a empresas (CGUTyP, 2016). Esto permite, retomar aptitudes que evidencien acciones estrategicas por parte de las y los estudiantes del nivel superior. Dentro del marco de referencia del propio Modelo educativo del SUUTT se encuentran, las Estadías Prácticas (EP), que están integradas en el mapa curricular de las Universidades Tecnológicas (UUTT) en los niveles de Técnico Superior Universitario (TSU) y en Licenciatura.

Es importante señalar, que la EP carece de una fundamentación adecuada, ya que no se define concretamente que es lo que se espera del estudiante y de la UPS, a fin de obtener datos significativos, estar de acuerdo a las características y necesidades del sector productivo y de servicios turísticos, específicamente en el desarrollo de productos gastronómicos. Por lo que, la EP se cursa en los últimos cuatrimestres de los niveles de TSU y de ingenierías favoreciendo la formación profesional 
de las y los estudiantes. Se establece dentro de sus lineamientos, la obligatoriedad de 600 horas práctica durante el periodo cuatrimestral de 6 to y 11vo para licenciatura o ingenieria. Cada Programa Educativo (PE) de las UUTT se identifican los perfiles de egreso, dando más auge a la realización de la EP. A lo que respecta, al proceso de evaluación de esos cuatro meses, que los y las estudiantes permanecen en la empresa, son observados por Asesores Externos (AE) asignado con frecuencia por el gerente de capacitación o gerente de recursos humanos. En el caso de la Universidad, designa a un Asesor Interno (Al) para el seguimiento de la EP. Sin embargo, la evaluación que se aplica a través de un formato que contiene seis criterios de evaluación. Con la finalidad, de calificar con una equivalencia numérica de 10 a 8, así como menor a 8 es no satisfactorio es reprobación de la EP.

Por lo expuesto anteriormente, la propuesta de esta investigación pretende responder y aportar información relevante de que competencias profesionales deberá demostrar, en este caso las y los estudiantes de Licenciatura en Gastronomía de la Universidad Tecnológica de Puebla. De acuerdo, con algunas inquietudes observadas durante el acompañamieto como Al de ambos niveles del PE en Gastronomía. Visto que, se describen algunas preguntas de investigación como son: ¿cuáles son las competencias profesionales, que la $y$ el estudiante de Licenciatura en Gastronomía debe demostrar en su desempeño de la Estadía Práctica; desde la perspectiva de los actores educativos involucrados?, ¿cuál es el marco de referencia de logros generales y específicos, durante la Estadía Práctica del estudiante de la licenciatura en Gastronomía, con relación a las competencias profesionales establecidas en el área de Alimentos y Bebidas? y ¿qué criterios de evaluación de las competencias profesionales deberán incluirse en un instrumento de evaluación en la Estadía Práctica, como referente del desempeño de los estudiantes de licenciatura en Gastronomía de la UTP?.

La revisión de literatura a fondo de los conceptos que deben incluirse en los instrumentos de evaluación y seguimiento de la EP, siendo el caso de la UTP, la cual carece de referentes teóricos, metodológicos y contextuales que desconocen los actores educativos de dicho proceso. Asimismo, la valoración insuficiente que compruebe el desempeño de las y los estudiante durante su EP debe interesarle a ambas partes, con la finalidad de lograr propuestas de mejora en el desarrollo de competencias profesionales precisas del entorno y de las tendencias culinarias que requiere los diferentes segmentos de mercado. Las UPS de la ciudad de Puebla colaboran de forma conjunta con las Universidades Tecnológicas del Estado para contruir redes de conocimiento ampliado, análisis situacional de los distintos sectores, las necesidades que requieren los clientes internos y externos de las organizaciones, entre otros factores que se consideran en un bienestar social armonióso y competitivo.

Con base, a las preguntas presentadas se redactan los objetivos de la investigación que se llevarán a cabo a través de la indagación exhaustiva, lo cual se redacta el objetivo general de la investigación es: diseñar un instrumento de evaluación con los niveles del desempeño óptimos para los y las estudiantes de Licenciatura en Gastronomía demostrando sus competencias profesionales en la Estadía Prática, de acuerdo a las características y necesidades de la organización. Sumándose los objetivos específicos como: analizar que competencias profesionales con base en el perfil de egreso de la Licenciatura en Gastronomía, son básicas para lograr una evaluación del desempeño, a través de la realización de su Estadía Práctica. Desde la perspectiva de los actores educativos y del sector productivo y de servicios involucrados. El segundo objetivo es Documentar un marco de 
referencia general y específico con relación a las competencias profesionales establecidas en el área de alimentos y bebidas, considerado para la realización de la Estadía Práctica de los y las estudiante de la licenciatura en Gastronomía. El tercer objetivo es aplicar un instrumento de evaluación con los criterios de desempeño del estudiante de la licenciatura en Gastronomía que evidencie las competencias durante la realización de la Estadía Práctica.

\section{DESCRIPCIÓN DE LA ESTRATEGIA METODOLÓGICA}

Para efectos de esta investigación, que busca determinar los niveles de desempeño de las competencias profesionales de los y las estudiantes de Licenciatura en Gastronomía de la UTP, se empleará un paradigma científico a la investigación cuantitativa donde se propone un problema de investigación, El tipo de investigación es exploratorio, utilizando como método el estudio de caso. Así como, la técnica de la observación estructurada con un cuestionario. No obstante, es indispensable referirnos a los y las estudiantes de licenciatura en Gastronomía realizando su EP, el diseño del instrumento de evaluación con procedimientos adecuados para su viabilidad y factibildiad.

Por otro lado, la perspectiva de la investigación es educativa donde aun se encuentran inscritos los y las estudiantes de la EP de licentiatura. La relevancia de la investigación, es que no hay un instrumento de evaluación que se aplique en la EP y que sea pertinente a las situaciones contextuales de cada establecimiendo de alimentos y bebidas $u$ otras organizaciones del sector turistico. Los resultados que se obtengan durante la aplicación del instrumento de evaluación en los momentos de EP, favorecerá para otras investigaciones.

\section{INSTRUMENTO DE EVALUACIÓN}

El instrumento de evaluación con su marco teórico referido al alineamiento constructivista de Jhon Biggs que favorecerá a la mejora de las recomendacio señaladas por el Consejo Nacional para la Calidad de la Educación Turística (CONAET) en su última visita a la UTP en el año 2018, con la sugerencia de fortalecer la trayectoria académica de los y las estudiantes de la licenciatura en Gastronomía, así como, identificar los niveles de desempeño en el que se encuentra el estudiante, para retomar las medidas pertinentes de revisión y actualización de los PE de la división académica de la UTP.

La construcción del instrumento de evaluación fue a través de un marco de referencia del MEUUTT, perfil de egreso del licenciado en Gastronomía y Tuning de América Latina, considerando los niveles de desempeño establecidos a través de las escalas de evaluación por competencias establecida en el PE de Gastronomía. Finalmente, se elaboró una rúbrica de observación del desempeño con corte cuantitativo que permitió evidenciar las competencias profesionales que los y las estudiantes desarrollan durante su formación profesional. Con el propósito de alcanzar logros autonómos y óptimos en la EP de los y las estudiantes de licenciatura en Gastronomía. Seguido, de la experiencia como Al con respcto a las acciones y comportamientos de los y las estudiantes al asistir a su EP. Se consideran algunos elementos indispensables que se consideran como parte de las variables, que integrará la rúbrica.

Para este procedimiento se realizó un esquema de las competencias profesionales indicadas para el PE de Gastronomía de manera general. Esto permitió que se seleccionará las variables principales que el y la estudiante de licenciatura en Gastronomía debe conocer y generar en el ámbito laboral. Se describen a continuación en la tabla 1. Como primera variable se indica el 
Desarrollo de concepts gastrómicos, donde el y la estudiantes son capaces de generar nuevos productos gastronómicos, que satisfaga a un segmento de mercado previamente estudiado a través de un estudio de investigación de mercados. La orientación de está variable, se basa de diversos procesos en el antes-durantes y despúes para crear e innovar un producto gastronómico con características que garantice y cumpla la expectativa de los clientes finales tanto internos como externos. Como segunda variable se encuentra la Administración de
Establecimientos de Alimentos y Bebidas, habilidad que se debe generar en todos los sentidos de producción, ejecución, integración de personal, supervisión de los procesos y materia prima, comunicación efectiva con proveedores, clientes internos y externos. Por último, la variable de Desarrollo de propuesta culinaria, la cual desarrollan ciertas habilidades administrativas y de técnicas culinarias propias de las diferentes áreas de cocina, correspondiente a: cocina caliente, cocina fría, parrillaje, salsas, panadería, repostería, entre otras, ya que puede variar al tipo de restaurante.

Tabla 1

\begin{tabular}{|c|c|c|}
\hline Descripción de Variables & & \\
\hline Variables & Definición Conceptual & Definición Operacional \\
\hline $\begin{array}{l}\text { 1.Desarrollo De Conceptos } \\
\text { Gastronómicos }\end{array}$ & $\begin{array}{l}\text { Evidenciar que el alumno } \\
\text { a través del diagnóstico } \\
\text { del potencial culinario, la } \\
\text { ingeniería de menús, cocina } \\
\text { mexicana e internacional } \\
\text { representativa y herramientas } \\
\text { financieras es capaz } \\
\text { de fortalecer al sector } \\
\text { gastronómico y contribuir al } \\
\text { desarrollo económico de la } \\
\text { zona. }\end{array}$ & $\begin{array}{l}\text { Dominio de habilidades } \\
\text { culinarias } \\
\text { Habilidades de ingeniería de } \\
\text { menú } \\
\text { Habilidades de toma de } \\
\text { decisiones, asertividad, } \\
\text { trabajo en equipo, liderazgo y } \\
\text { pensamiento crítico }\end{array}$ \\
\hline $\begin{array}{l}\text { 2.Administración de } \\
\text { establecimientos de Alimentos } \\
\text { y Bebidas }\end{array}$ & $\begin{array}{l}\text { El proceso administrativo son } \\
\text { las actividades para que el } \\
\text { estudiante logre las metas } \\
\text { organizacionales a través de } \\
\text { los objetivos particulares para } \\
\text { la supervisión y cumplimiento } \\
\text { de estándares y reglamentos } \\
\text { del área de gastronomía. }\end{array}$ & $\begin{array}{c}\text { Habilidades de comunicación } \\
\text { Habilidades en TIC } \\
\text { Habilidades en administración } \\
\text { Toma de decisiones } \\
\text { Herramientas de control } \\
\text { Técnicas de supervisión } \\
\text { Relaciones interpersonales }\end{array}$ \\
\hline $\begin{array}{l}\text { 3. Desarrollo de propuestas } \\
\text { culinarias }\end{array}$ & $\begin{array}{l}\text { Uso de métodos que permiten } \\
\text { la demostración de la } \\
\text { habilidad culinaria }\end{array}$ & $\begin{array}{c}\text { Métodos } \\
\text { Técnicas culinarias } \\
\text { Creatividad } \\
\text { Liderazgo }\end{array}$ \\
\hline
\end{tabular}

Fuente: Elaboración propia 


\section{VALIDEZ Y FACTIBILIDAD}

La construcción del instrumento de evaluación, con base a las tres variables mencionadas anteriormente se contó con la participación de 31 estudiantes del grupo $10^{\circ}$ cuatrimestre grupo "A" de la División Académica de Gastronomía de la UTP, se aplicó el instrumento en los primeros días del mes de noviembre de 2018, con relación a 31 items que integraba la rúbrica de observación de desempeño como prueba piloto y así proceder a la validez de dicho instrumento. Una vez finalizada la aplicación, fue entregada en tiempo y forma para el inicio del procesamiento de la información relacionada con las respuestas obtenidas. Este tipo de estudio exploratorio carece de información y es poco investigado, por lo cual se deben tomar precauciones más rigurosas para investigaciones posteriores, a ser más flexibles en la metodología que sea recurrida en comparación con otros tipos de estudio.

Al momento, de la recolección de datos se trabajo con una hoja de calculo en formato excel para recabar la información de forma ordenada, con las columnas y filas identificando las respuestas. El siguiente paso es la creación de una base de datos, en el software SPSS (Statistical Package for the Social Sciences) que es un programa estadistico que permite validar y adquirir la confiabilidad del intrumento de evaluación. Por lo consiguiente, se migraron los datos de excel al SPSS para correr el progama estadistico con base caracteristicas especificas para la correlación de las preguntas, de la prueba piloto. El procedimiento para la validación y factibilidad se sustenta en la estadística inferencial, con la interpretación de resultados de la aplicación del instrumento y extraer conclusiones.

El registro de datos estadísticos en la investigación permitió que se analizará de forma objetiva y se identificará una serie de elementos que favorecen a tomar decisiones. Se determinó la consistencia o la estabilidad de las medidas cuanto el proceso de medición se repite con otros sujetos, a esto se le llama fiabilidad (Haertel, 2006). Asimismo, la confiabilidad según la teoría clásica, se define como el grado en que el instrumento de varios ítems mide consistentemente muestra de la población.

La medición consistente se refiere al grado en que una medida está libre de errores. El coeficiente de confiabilidad se expresa con la letra $r$ e indica la fuerza de la asociación. El valor $r$ varía entre $-1 \mathrm{y}+1$, un valor de 0 indica que no existe relación entre los dos puntajes, mientras que un valor cercano a -1 o $a+1$ indica una relación muy cercana, negativa o positiva, respectivamente. Un valor positivo indica que las personas con puntaje alto en una primera aplicación de la escala también puntuarán alto durante la segunda ocasión. Una confiabilidad negativa indica un error en el cálculo o una terrible inconsistencia de la escala. El error se conceptualiza como la diferencia entre el valor observado y el verdadero o un valor promedio de las mediciones repetidas. Sin embargo, por la imposibilidad teórica de calcular directamente la varianza de la puntuación verdadera, la ecuación fue modificada, de tal suerte que la varianza de la puntuación verdadera es igual a 1 menos la varianza del error (Oviedo \& Campos Arias, 2005).

Los instrumentos que buscan medir un constructo pueden ser validados en forma indirecta basándose en la relación que muestren los ítems que componen la escala; es decir, presentan una excelente consistencia interna o interrelación entre las preguntas o incisos que hacen parte de la escala. Esto es realizar una validación de un constructo sin un patrón de referencia. El coeficiente alfa fue descrito en 1951 por Lee J. Cronbach. Es un índice usado para medir la confiabilidad del tipo consistencia interna de una escala, es decir, para evaluar la magnitud en que los ítems de un instrumento están correlacionados. En otras 
palabras, el alfa de Cronbach es el promedio de las correlaciones entre los ítems que hacen parte de un instrumento. También se puede concebir este coeficiente como la medida en la cual algún constructo, concepto o factor medido está presente en cada ítem. La forma más sencilla de calcular el valor del alfa de Cronbach es multiplicar el promedio de todas las correlaciones observadas en los ítems por el número de ítems que componen una escala, y luego dividir el producto entre el resultado de la suma de 1 más el producto de la multiplicación del promedio de todas las correlaciones observadas por el resultado de la resta de 1 al número de items: $\{a=n . p / 1+p(n-1)\}$, donde $\mathrm{n}$ es el número de ítems y $\mathrm{p}$ es el promedio de todas las correlaciones

El instrumento de evaluación obtuvo una fiabilidad de 0.894 con referencia al Alfa de Cronbach, por lo que resulta confiable para su estudio. La eficiencia de esta prueba es del $95 \%$ para muestras pequeñas y a través del software del SPSS, se comprobó cada uno de los ítems de la rúbrica de observación de desempeño para su aplicación en dos momentos del periodo cuatrimestral. Asimismo, analizar los resultados de la evaluación inicial y final, utilizando el método estadístico no probabilístico de Wilcoxon para conocer el nivel de significancia que señalan a continuación en la tabla 2 donde se indican solo algunos ítems. Estadísticamente se visualiza en la tabla 2 , que la prueba del nivel de significancia sirvió para comparar las dos muestras de datos recogidas antes y después de los mismos sujetos, cuyo valor central fue cero. Las diferencias iguales a cero se eliminan y el valor absolutos de las desviaciones con base al valor central, son ordenadas de menor a mayor. Los datos idénticos se les asignan un lugar medio de la serie.

La suma de los rangos se realizó por separados, signos positivos y los negativos. Con base al nivel de significancia que se establece para las ciencias sociales es de 0.05. En este sentido, se comprobó que si hubo un nivel de significancia entre la primera aplicación de la guía de observación de desempeño y el segundo momento de la aplicación de la evaluación. Sin embargo, en los tres últimos ítems que establece la parte de la gestión administrativa y financiera carece de significancia, deduciendo que las habilidades gerenciales y financieras deben fortalecerse en el proceso de enseñanza aprendizaje. 
Tabla 2

Pantalla De SPSS. Resultados De Prueba De Algunas Variables, Con Método De Wilcoxon

\begin{tabular}{|c|c|c|c|c|}
\hline \multicolumn{5}{|c|}{$\underline{\text { Rangos }}$} \\
\hline & & $\mathrm{N}$ & $\begin{array}{c}\text { Rango } \\
\text { Promedio }\end{array}$ & $\frac{\text { Suma de }}{\underline{\text { Rangos }}}$ \\
\hline \multirow{4}{*}{$\begin{array}{l}\text { Analiza el segmento de } \\
\text { mercado - Analiza el } \\
\text { segmento de mercado }\end{array}$} & Rangos negativos & $0^{d}$ & .00 & .00 \\
\hline & Rangos positivos & $9^{e}$ & 5.00 & 45.00 \\
\hline & Empates & $12^{f}$ & & \\
\hline & Total & 21 & & \\
\hline \multirow{2}{*}{$\begin{array}{l}\text { Indica tareas específicas - } \\
\text { Indica tareas especificas }\end{array}$} & Rangos negativos & $0^{\mathrm{m}}$ & .00 & .00 \\
\hline & $\begin{array}{l}\text { Rangos positivos } \\
\text { Empates } \\
\text { Total }\end{array}$ & $\begin{array}{l}12^{n} \\
9^{\circ} \\
21\end{array}$ & 6.50 & 78.00 \\
\hline \multirow{2}{*}{$\begin{array}{l}\text { Asigna el capital humano - } \\
\text { Asigna el capital humano }\end{array}$} & Rangos negativos & $0^{p}$ & .00 & .00 \\
\hline & $\begin{array}{l}\text { Rangos positivos } \\
\text { Empates } \\
\text { Total }\end{array}$ & $\begin{array}{l}10^{\mathrm{q}} \\
11^{\mathrm{r}} \\
21\end{array}$ & 5.50 & 55.00 \\
\hline \multirow{2}{*}{$\begin{array}{l}\text { Diseña de la propuesta - } \\
\text { Diseña de la propuesta }\end{array}$} & Rangos negativos & $0^{\mathrm{ab}}$ & .00 & .00 \\
\hline & $\begin{array}{l}\text { Rangos positivos } \\
\text { Empates } \\
\text { Total }\end{array}$ & $\begin{array}{l}10^{\mathrm{ac}} \\
11^{\mathrm{ad}} \\
21\end{array}$ & 5.50 & 55.00 \\
\hline \multirow{2}{*}{$\begin{array}{l}\text { Aplica la tendencia culinaria } \\
\text { - Aplica la tendencia } \\
\text { culinaria }\end{array}$} & Rangos negativos & $0^{\text {ae }}$ & .00 & .00 \\
\hline & $\begin{array}{l}\text { Rangos positivos } \\
\text { Empates } \\
\text { Total }\end{array}$ & $\begin{array}{l}12^{\text {af }} \\
9^{\text {ag }} \\
21\end{array}$ & 6.50 & 78.00 \\
\hline \multirow{3}{*}{$\begin{array}{l}\text { Diseña menús } \\
\text { estandarizados - Diseña } \\
\text { menús estandarizados }\end{array}$} & Rangos negativos & $0^{\text {aq }}$ & .00 & .00 \\
\hline & Rangos positivos & $11^{\mathrm{ar}}$ & 6.00 & 66.00 \\
\hline & $\begin{array}{c}\text { Empates } \\
\text { Total }\end{array}$ & $\begin{array}{c}10^{\text {as }} \\
21\end{array}$ & & \\
\hline \multirow{8}{*}{$\begin{array}{l}\text { Aplica protocolos de } \\
\text { servicio y técnicas de } \\
\text { montaje - Aplica protocolos } \\
\text { de servicio y técnicas de } \\
\text { montaje } \\
\text { Muestra interés e iniciativa - } \\
\text { Muestra interés e iniciativa }\end{array}$} & Rangos negativos & $0^{\mathrm{bl}}$ & .00 & .00 \\
\hline & Rangos positivos & $13^{\mathrm{bm}}$ & 7.00 & 91.00 \\
\hline & Empates & $8^{\mathrm{bn}}$ & & \\
\hline & Total & 21 & & \\
\hline & Rangos negativos & $0^{\text {bo }}$ & .00 & .00 \\
\hline & Rangos positivos & $16^{\mathrm{bp}}$ & 8.50 & 136.00 \\
\hline & Empates & $5^{\mathrm{bq}}$ & & \\
\hline & Total & 21 & & \\
\hline
\end{tabular}




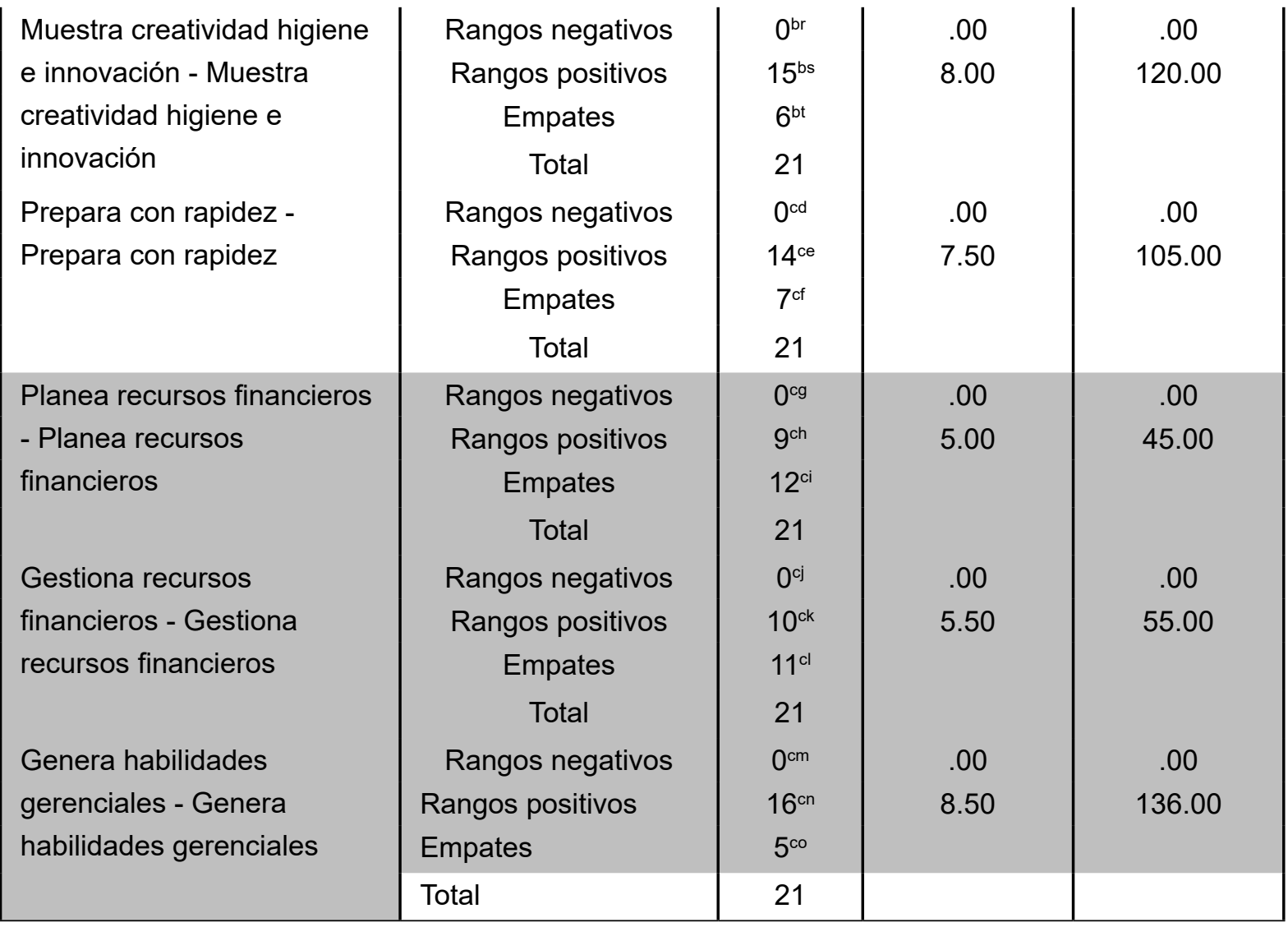

Fuente: Elaboración propia

\section{CONCLUSIONES}

La Organización de Estados Iberoamericanos (OEI), el Fondo Monetario Internacional (FMI), la Organización para la Cooperación y el Desarrollo Económico (OCDE), el Banco Interamericano de Desarrollo (BID), la UNESCO y el Fondo de las Naciones Unidades para la Infancia (UNICEF) establecen el Marco de Análisis y Sugerencias, en el marco de la calidad de la Educación, en el que se responsabilizan a la escuela y a sus profesores para el éxito de la misma, con base específicamente en el actuar de la planta docente y con relación a una de las múltiples dimensiones de la calidad de la UNESCO, que se encuentra en el desarrollo de actividades educativas con el propósito de orientar los procesos educativos, para promover el desarrollo de competencias en las y los estudiantes de las IES. Por lo anterior, se presentan grandes retos con relación a la profesionalización de la enseñanza en la Educación Superior, los cuales, demandan dejar a un lado el individualismo docente para asumir: un trabajo en equipo por proyectos responsables, comprometidos, con autonomía, atención de la diversidad, énfasis en la pluralidad de instrumentos, herramientas y tecnologías así como de situaciones de aprendizaje, conocimientos y saberes que potencialicen el aprendizaje para la vida. ${ }^{4}$ El Modelo Educativo de las Universidades Tecnológicas con enfoque por competencias aparece como una opción para resolver la demanda de la sociedad del conocimiento y obliga a replantear lo que comúnmente se ha considerado como formación; el modelo educativo considera algunos principios en los que destacan: el aprendizaje significativo, la transferencia en contextos diferentes, la polivalencia, el aprendizaje como

\footnotetext{
$4 \quad$ Meirieu (1989) en Perrenoud P. (2007). Diez nuevas competencias para enseñar. Invitación al viaje, Graõ, Colofón, México, 168 págs.
} 
proceso, alternancia en la formación y criterios de desempeño (Quiroz, 2010).

Las y los estudiantes competentes en el desarrollo de conceptos gastronómicos, en las propuestas culinarias innovadoras para dirigir el producto y servicio gastronómico a segmentos de mercados apropiados. El proceso educativo del estudiante de licenciatura de Gastronomía no concluye en el entorno áulico, sino a partir del acompañamiento y retroalimentación de la Estadía Práctica alineado al propósito, a las competencias profesionales a desarrollar en un área específica de su profesión. Con el fin de que, alcance desempeños autónomos para solucionar problemas reales y tomar decisiones más asertivas en el Sector Turístico. Con el beneficio, de mejorar el mercado gastronómico con personal capacitado y orientado a la mejora continua de sus servicios y demandas que sugieren los clientes internos y externos de cada organización de alimentos y bebidas.

Por lo tanto, se considera que es indispensable orientar el propósito del proceso de evaluación en la EP de los estudiantes de licenciatura en Gastronomía. Con el sentido de alinear el propósito de la misma con las competencias profesionales a demostrar en cualquier área asignada por el sector productivo, en este caso por el Establecimiento de Alimentos y Bebidas. Si bien las competencias profesionales que se construyeron a través de un análisis epistemológico, metodológico permiten visualizar el nivel de logro de los estudiantes, al ejecutar cualquiera de las tareas encomendadas por un Gerente, un Chef Ejecutivo o tal vez, el mismo estudiante establezca sus propias decisiones como se demuestra en algunos resultados.

\section{RECOMENDACIONES}

EI MEUUTT con enfoque por competencias debe ser revisado de forma más integral por cada uno de los actores educativos que la integran a fin de alinear el Modelo Pedagógico de la UTP con el proceso de enseñanza aprendizaje, articulando el sentido más amplio de la Estadía Práctica. De este modo se evita que la Estadía Práctica se convierta un requisito más del proceso de titulación. Con los resultados obtenidos, permite realizar otras investigaciones posteriores a la evaluación del estudiante.

La Alineación Constructivista favorece al MEUUTT en la construcción de aprendizajes idóneos para los estudiantes de la Licenciatura en Gastronomía y la oportunidad de colaborar con otras divisiones académicas. Es decir, los resultados de evaluación de las Estadías Prácticas se pueden analizar e interpretar para estudios posteriores con la finalidad de reconocer los niveles de desempeño de los estudiantes y su impacto en el Sector Turístico, en áreas de alimentos y bebidas. En conjunto, los actores del proceso educativo y la vinculación con el Sector Turístico es indispensable para lograr acuerdos y estrategias en beneficio de la sociedad. Con miras a, un futuro inmediato en el segmento de mercado gastronómico del estado de Puebla, México. Cambiando la mirada, de un tipo de turismo religioso a un turismo gastronómico.

En consecuencia, la investigación fue cumpliendo con cada uno de sus objetivos relacionados con documentar con el marco de referencia general y específico con relación a las competencias profesionales establecidas en el área de alimentos y bebidas, considerado para la realización de la Estadía Práctica del estudiante de la licenciatura en Gastronomía, el análisis de los niveles de desempeño que la y el estudiante de licenciatura en Gastronomía debe demostrar en el sector de servicios, con la aplicación de la rúbrica de observación de desempeño. Esa rúbrica, fue diseñada con base a la investigación antes mencionada, donde se observó el antes y después de las y los estudiantes que acudieron a su EP en el periodo de enero abril 2019 en establecimientos de A y $B$ de la ciudad de 
Puebla a través de las y los Asesores Externos, a quienes se les explico la evaluación que tenían que realizar. Como recomendación, es indispensable que en el primer acercamiento con las y los Asesores Externos se realice una capacitación programada por parte de la división académica, como primera fase del proceso de EP con el propósito de: conocer con anterioridad el instrumento de evaluación, cuales son los momentos de la aplicación, los objetivos de la evaluación, quienes serán las y los Asesores Internos, cuáles serán los medios de comunicación, establecer mecanismos de colaboración, entre otros. Los resultados que se obtuvieron durante la aplicación, que fueron enunciados en el capítulo correspondiente de esta investigación fueron meramente descriptivos. Cabe mencionar, que la intensión de observar el nivel alcanzado por la y el estudiante de licenciatura en Gastronomía fomenta parte de una cultura de un proceso de evaluación, el cual debe existir una retroalimentación en el sentido de que manera impacta en la sociedad, los egresados.

El trabajo de investigación promueve la realización de futuras aproximaciones realizadas a beneficio de la Estadía Práctica, un proceso de evaluación que ha sido retomado como un requisito administrativo para la titulación de las y los estudiantes. Por su parte la investigación pretende además de documentar de forma conceptual y metodológica algunos contenidos que posteriormente podrían retomarse en el MEUUTT. La investigación documental presentada, específicamente podría considerar:

- Fundamentar teóricamente el alineamiento constructivista con el proceso de la Estadía Práctica, a través de las observaciones realizadas se mostró que la utilización de un instrumento de evaluación se podría medir los niveles de desempeño de las y los estudiantes para tomar decisiones por parte de los diversos actores educativos que participan en el proceso de enseñanza aprendizaje.

- Establecer desde el enfoque por competencias la participación de las y los asesores de la EP a través de capacitaciones cuatrimestrales programadas por cada PE. Con la utilización del uso y manejo de las $\mathrm{TIC}$, estrategias que se requieran para la EP, motivación y promoción del desarrollo de las competencias profesionales del licenciado en Gastronomía.

- Presentar la teoría del Alineamiento Constructivista como parte fundamental del MPUTP con relación a la formalización operativa. El instrumento de evaluación responde al interés de un solo PE, sin embargo, no descarta la posibilidad de rediseñarse para otros PE de la UTP.

- $\quad$ Contrastar la evaluación inicial y final pero de los niveles académicos del MEUUTT, siendo el TSU y Licenciado en Gastronomía. En los periodos cuatrimestrales correspondientes a la Estadía Práctica. Con el objetivo de identificar los niveles de desempeño alcanzados como TSU y como licenciado para la formación profesional que se espera del PE y su impacto en la sociedad.

\section{REFERENCIAS BIBLIOGRÁFICAS}

- $\quad$ Adler, F. (1964). Positivism. Nex York, Estados Unidos de Norteamérica: The Free Press.

- Cázares, A., \& Cuevas, d. I. (2007). Planeación y Evaluación basadas en Competencias. México: Trillas.

- Cerda, H. (1991). Capítulo 7: Medios, instrumentos, Técnicas y Métodos de Recolección de Datos de Información. Unidad Curricular: Metodología de la Investigación II. Caracas, Venezuela, Bogotá, Venezuela: Universidad Abierta y a Distancia. 
- $\quad$ CGUTyP. (2016). MODELO EDUCATIVO Y METODOLOGÍA PARA EL DISEÑO CURRICULAR BASADO EN COMPETENCIAS PROFESIONALES. Recuperado el 12 de septiembre de 2016, de Coordinación General de Universidades Tecnológicas y Politécnicas: http://cgut.sep.gob.mx/

- $\quad$ Chetty, S. K. (1996). The case study method for research in small- and medium - sized fi rms. International small business journal,. International of Education, 5.

- CONOCER. (23 de Enero de 2017). Secretaría de Educación Pública. Obtenido de Secretaría de Educación Pública: http://www.conocer.gob.mx/index.php?option=com_ content\&view=category\&layout=blog\&id=1\&ltemid=3

- D`Ancona, C. (1996). Metodología cuantitativa Estrategias y Técnicas de Investigación Social. España.

- Díaz, B. Á., \& Luna, M. A. (2014). Metodología de la Investigación Educativa. México: Díaz de Santos.

- Duran, M. (2002). Marco Epistemológico de la enfermería. Aquichan.

- Escobar, H. G. (2014). La Evaluación del Aprendizaje, su Evaluación y elementos en el marco de la Formación Integral. Obtenido de: Universidad Católica de Manizales

- Fernández, P. A., Alarcón, P. M. \& Barajas, A. G.(2018). Programa de Estudios Seminario de Tesis III: Contexto y Teoría

- García, C. B. Modelos Teóricos e Indicadores de Evaluación Educativa. Recuperado de: www. sinectica.iteso.mx

- Haertel, E. (2006). Educational Measurenment. American Council on Education and Praeger Publishers, 65-110.

- Hernández, S., Fernández, C. C., \& Baptista, L. P. (2004). Metodología de la Investigación. México: Mac Graw Hill.

- Huerta, A. J., Pérez, G. S. \& Castellanos, C. A. (2018). Desarrollo Curricular por Competencias Profesionales integrales. Recuperado de: http://educacion.jalisco.gob.mx/ consulta/educar/13/13Huerta.html

- Landero, H. R., \& González, R. M. (2016). Estadística con SPSS y Metodología de la Investigación. México: Trillas.

- López, F. B., \& Hinojosa, K. E. (2016). Evaluación para el Aprendizaje. Alternativas y nuevos desarrollos. México: Trillas.

- OCDE. (2011). Establecimiento de un Marco para la evaluación e incentivos para docentes. México, Pág.63: Secretaria General de OCDE.

- OIT. (22 de Enero de 2016). Competencias para el Empleo. Obtenido de Competencias para el Empleo: http://www.ilo.org/skills/pubs/WCMS_180580/lang--es/index.htm 
- Oviedo, C., \& Campos Arias, A. (2005). Aproximación al uso del coeficiente alfa de Cronbach. Revista Colombiana de Psicatria , 572-580.

- Quiroz, L. M. (2010). Modelos Educativos en el IPN y el ITESM. Las competencias profesionales en la educación superior. México: ANUIES.

- Tacca, H. D. (2011). El „Nuevo Enfoque Pedagógico: Las Competencias“. Investigación Educativa, 167.

- Tamayo, M. (2004). El Proceso de la Investigación Científica. México: Limusa.

- $\quad$ Tejada, F. J. (1999). Acerca de las competencias profesionales.

- Tobón, S. (2006). Aspectos Básicos de la Formación Basada en Competencias. Talca: Proyectos Mesesup, 10-14.

- UTP, U. T. (28 de agosto de 2012). Reglamento Escolar. SEP, págs. 1-4. 